\title{
Evaluation of the electrodynamic forces in high-speed permanent magnet machines with rotor eccentricity
}

\author{
Corentin DUMONT*, Adrien GILSON**, Virginie KLUYSKENS*, Christophe ESPANET ${ }^{* *}$ and \\ Bruno DEHEZ* \\ * Department of Mechatronic, Electrical Energy, and Dynamic Systems (MEED), Université catholique de Louvain \\ Place du Levant 2, Louvain-la-Neuve 1348, Belgium \\ E-mail: corentin.dumont@uclouvain.be \\ ${ }^{* *}$ Energy Department, FEMTO-ST Institute, University of Franche-Comté \\ Avenue Jean Moulin 2, Belfort 90000, France
}

Received: 2 February 2017; Revised: 2 May 2017; Accepted: 13 June 2017

\begin{abstract}
In permanent magnet motors, the presence of rotor eccentricities can alter the airgap field distribution. This results in parasitic radial detent forces that can be reduced by connecting the stator phases in parallel. As a consequence, currents are passively induced in the windings when the rotor spins in an off-centered position, yielding balancing electrodynamic forces. Specific models were developed to predict these forces, but their complexity can be prohibitive. Therefore, this paper proposes to study the effect of the rotor off-centering in permanent magnet motors using a simpler model developed for electrodynamic bearings. This model consists in a linear differential equation with only four parameters that depend neither on the spin speed nor on the rotor position. As an illustration, the paper applies this model to the study of a high-speed, slotted permanent magnet motor. To support this, the main hypotheses of the model are validated in this particular case. Then, the centering electrodynamic forces in a staticeccentricity configuration are predicted using the model and compared to finite element simulation results. Finally, a preliminary study showing the impact of the width and permeability of the stator teeth on the centering force is performed.
\end{abstract}

Keywords : Synchronous, Motor, Eccentricity, Force, Passive, Electrodynamic, Model

\section{Introduction}

Bearing wear and manufacturing tolerances can lead to rotor eccentricities in permanent magnet (PM) machines. This affects the symmetry of the field distribution in the airgap, thereby creating a radial detent force known as unbalanced magnetic pull (UMP) (Rahideh and Korakianitis, 2011) (Dorrell et al., 2009). The UMP is a potential source of excessive noise, vibrations, and additional wear of the mechanical bearings (Kim and Lieu, 2005) (Li et al., 2007). Therefore, passive and active strategies have been studied to reduce these effects. Among them, the parallel connection of the stator windings is well known (Burakov and Arkkio, 2007) (Kasten and Redemann, 2014). It allows passively induced currents to flow in the stator windings when the rotor spins in an off-centered position, thereby creating forces that balance the UMP. Different models predicting the balancing forces in permanent magnet (PM) motors have been proposed (Burakov and Arkkio, 2006) (Dorrell and Ionel, 2012). These models can be adapted to a wide range of machines. However, they include assumptions on the rotor motion so that only static and dynamic eccentricities are considered, and the model parameters must be re-estimated if the operating point of the motor changes.

The operation of heteropolar electrodynamic bearings (EDBs) is also based on passively induced centering forces. In particular, the origin of these forces is the same as in PM motors with parallel winding connections. When the rotor is off-centered, the PM field distribution changes in the air gap, thereby creating additional harmonics that increase with the off-centering (Rahideh and Korakianitis, 2011). If the main harmonics in the initial PM field distribution has one pair of poles, the most significant harmonic created by the off-centering has two pole pairs. On the one hand, these harmonics 
have a negative effect since their interaction yields the UMP (Chiba et al., 2005). On the other hand, the two pole pairs harmonic can be linked by a winding that also has two pole pairs, thereby inducing balancing currents which tend to restore the centered position of the rotor (Dumont et al., 2014). Similarly, in the case of a one pole pair PM motor, the parallel connection of each motor phase may allow for the existence of conducting paths with two pole pairs and passive centering forces. Recent advances in the modeling of EDBs lead to the derivation of a linear equation predicting the dynamics of the bearing rotor (Dumont et al., 2016). This model includes no assumption on the rotor motion and spin speed. It depends on only four parameters that are identified through a limited number of magnetoquasistatic simulations.

In this context, this paper first proposes a method for predicting the passive electrodynamic forces in brushless PM motors using the model developed for heteropolar EDBs (Dumont et al., 2016). This model is then applied in the practical case of a high-speed DC motor dedicated to air compression that is currently being designed at the FEMTO-ST institute (Gilson et al., 2015). For example, the model could be used to investigate the presence of radial forces due to the use of air bearings that allows for small radial displacements of the rotor.

The paper is organized as follows. In section 2, the motor topology and winding connections are introduced. In section 3, the EDB model is presented and applied to the motor case. In section 4, the main hypotheses of the model are validated and the parameters corresponding to the study case are identified. Then, the model is exploited to predict the radial forces on the off-centered rotor in section 5. Finally, a preliminary study is performed, highlighting the impact of the stator slots width and permeability on the radial forces.

\section{Motor and phases connection}

Figure 1a shows the topology of the motor used as an example in this paper. This motor is designed for high-speed applications $(80 \mathrm{krpm})$ such as air compression. Therefore, it has a large airgap and a retaining sleeve around the PMs. The parameters of the motors are given in Tables 1 and 2. The motor active length is denoted $L$, and the nominal airgap is denoted $g=R_{s}-R_{s l}$.

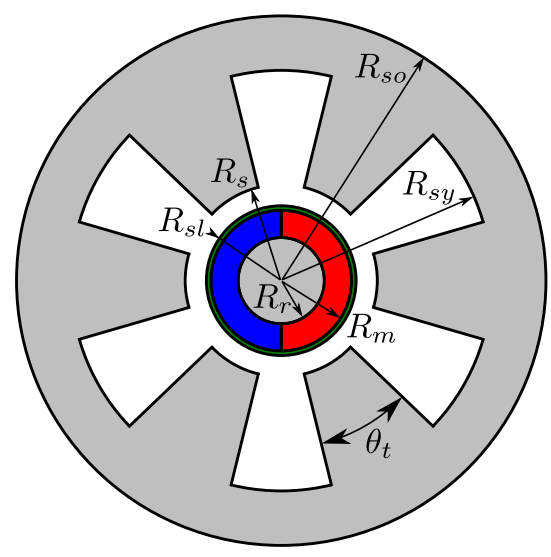

(a)

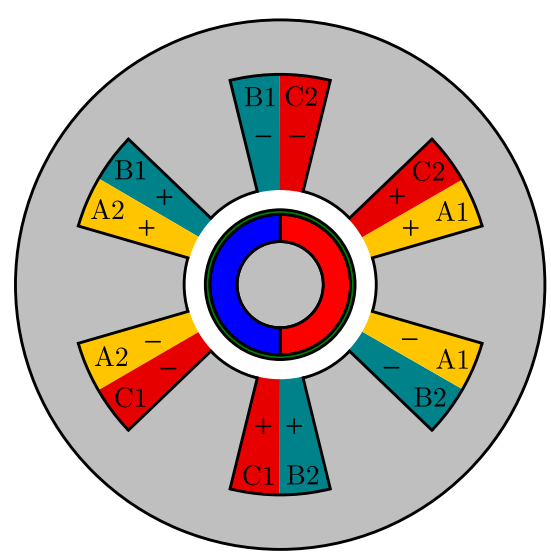

(b)

Fig. 1 Motor topology and phases arrangement. (a) Geometrical parameters of the motor. The internal rotor comprises surface-mounted PMs with one pole pair and a retaining sleeve. (b) The winding has three phases composed of two concentrated coils. For example, the coils A1 and A2 constitute phase A.

Table 1 Motor geometrical parameters.

\begin{tabular}{l|l}
\hline Parameter & Value \\
\hline$R_{s o}$ & $35[\mathrm{~mm}]$ \\
$R_{s y}$ & $27.8[\mathrm{~mm}]$ \\
$R_{s}$ & $12.7[\mathrm{~mm}]$ \\
$R_{s l}$ & $9.9[\mathrm{~mm}]$ \\
$R_{m}$ & $9.3[\mathrm{~mm}]$ \\
$R_{r}$ & $5.7[\mathrm{~mm}]$ \\
$\theta_{t}$ & $32.4\left[^{\circ}\right]$ \\
$L$ & $30[\mathrm{~mm}]$ \\
\hline
\end{tabular}

Table 2 Other motor parameters.

\begin{tabular}{l|l}
\hline Parameter & Value \\
\hline Remanent field density of the PM & $1.2[\mathrm{~T}]$ \\
Copper conductivity $\sigma_{c}$ & $6 e 7[\mathrm{~S} / \mathrm{m}]$ \\
Slot fill factor $r$ & 0.5 \\
Relative permeability of the PMs, retaining sleeve, and winding & 1 \\
Relative permeability of the rotor shaft and stator yoke & 1000 \\
Number of wire turns around each slot $N$ & 10 \\
\hline
\end{tabular}

The stator yoke has six slots and three phases, each of them being composed of two concentrated winding coils. As 
shown in Fig. 2, the coils can be connected in series or in parallel. In this paper, only the parallel connection is considered as it allows for passively induced currents to flow in the winding paths shown by the arrows in Fig. 2a. These paths have two pole pairs and are referred to as the suspension phases in the next sections.

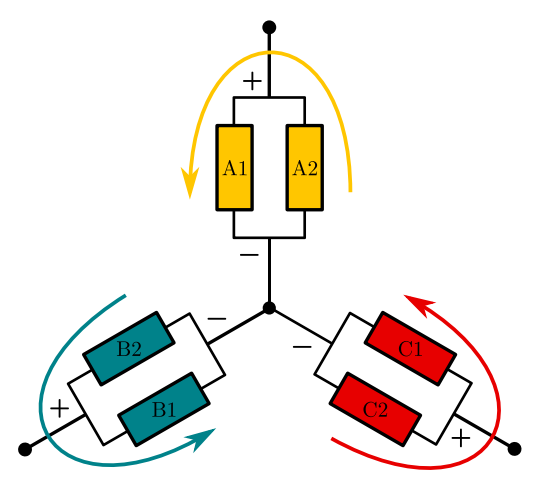

(a)

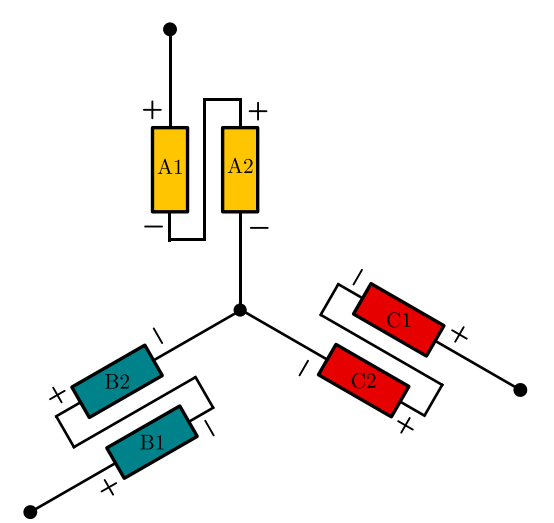

(b)

Fig. 2 Two different motor phase coils connections. (a) Phase coils connected in parallel. The arrows show the short-circuited current paths for the suspension currents. (b) Phase coils connected in series. There are no possible current paths in the windings for the suspension currents.

\section{Modeling}

As shown in (Kluyskens et al., 2017), the passive suspension forces can be studied independently from the motor function. This section focuses on the modeling of the passive radial forces, whereas more information about the motor control can be found in (Gilson et al., 2015).

Initially, this motor was designed for high-speed applications without considering a rotor off-centering. However, in case of rotor off-centering, passively induced currents flow in the motor phases that are connected in parallel, resulting in balancing forces. These passive electrodynamic forces can be predicted using the model initially developed for EDBs (Dumont et al., 2016). This model links the radial forces to the rotor position through a linear equation with constant coefficients:

$$
\dot{F}=-\frac{R}{L_{c}} F-j \omega F-K_{d} \dot{z}-\frac{3 K_{\Phi}^{2}}{2 L_{c}} \dot{z}-\frac{R K_{d}}{L_{c}} z-j \omega z K_{d}-j \omega z \frac{3 K_{\Phi}^{2}}{2 L_{c}},
$$

where the forces and rotor displacements are expressed in the stator frame using the complex notations $F=F_{x}+j F_{y}$ and $z=x+j y=|z| e^{j \phi}$, respectively. The parameters are gathered in Table 3. $R$ is the phase resistance of a suspension phase, $K_{d}$ is the negative stiffness associated with the detent force between the PMs and the stator yoke, and $K_{\Phi}$ is the PM flux constant defined in hypothesis 8 below. The cyclic inductance is $L_{c}=L-M$, where $L$ and $M$ are the self- and mutual inductances of the suspension phases, respectively.

Table 3 Model parameters and units.

\begin{tabular}{l|l|l}
\hline Parameter & Unit & Definition \\
\hline$R$ & $\Omega$ & Suspension phase resistance \\
$K_{d}$ & $\mathrm{~N} / \mathrm{m}$ & Detent stiffness \\
$K_{\Phi}$ & $(\mathrm{Ns} \Omega / \mathrm{m})^{1 / 2}$ & Flux constant \\
$L_{c}$ & $\mathrm{H}$ & Suspension phase cyclic inductance \\
$\omega$ & $\mathrm{rad} / \mathrm{s}$ & Spin speed \\
\hline
\end{tabular}

The model was derived under the following assumptions:

(1) the rotor spin speed $\omega$ is an input of the system and is constant;

(2) only translational eccentricity is considered, i.e., the magnetic axis of the rotor and winding remain parallel;

( 3 ) the materials have linear magnetic characteristics and therefore magnetic hysteresis and saturation are neglected;

(4) there is no proximity or skin effect in the conductors;

( 5 ) the eddy currents are neglected in the PMs, in the sleeve and in the stator yoke;

(6) the impact of the rotor off-centering $|z|$ on the winding inductances is neglected; 
( 7 ) the detent force $F_{d}$ between the PMs and the yoke obeys: $F_{d}=-K_{d} z$, where the detent stiffness $K_{d}$ is real and negative;

( 8 ) the PM flux linked by a suspension phase $\Phi_{M k}$ obeys: $\Phi_{M k}=|z| K_{\Phi} \cos \left(\omega t+\phi+\frac{2 \pi(k-1)}{3}\right)$, where $k \in\{1,2,3\}$ is the phase number;

(9) the motor currents do not impact the radial forces on the rotor.

Although the present model aims at predicting the effects of the rotor eccentricities, the impact of the eccentricity on the winding inductances is neglected through assumption 6. Making this assumption not only allows to obtain a model that is simple, it is valid in the present case because of to the large PM thickness. This large thickness results in the ferromagnetic rotor shaft being far from the stator teeth. As a result, the impact of a small rotor eccentricity on the winding current field is negligible. This is validated in section 4.

Finally, let us note that the slot geometry and fill factor are constant, see section 2. Therefore, the coefficients $K_{d}$, $K_{\Phi}^{2} / L_{c}$, and $R / L_{c}$ in Eq. (1) do not depend on the number of winding turns $N$. Therefore, $N$ has no impact on the forces and can be chosen considering only the constraints associated with the motor function.

\section{Parameters identification and model validation}

In this section, the parameters from Eq. (1) are evaluated considering the motor geometry and parameters presented in section 2, and the hypotheses 6-9 of the model are validated using two-dimensional magnetoquasistatic finite element (FE) simulations.

\subsection{Suspension phase resistance}

The suspension phase resistance is obtained without taking the end-windings into account. Considering the motor parameters from Table 1 and 2, this yields:

$$
R=\frac{48 L N^{2}}{\sigma_{c} r \pi\left(R_{s y}^{2}-R_{s}^{2}\right)\left(1-\frac{\theta_{t}}{60}\right)}=5.4[\mathrm{~m} \Omega] .
$$

\subsection{Radial detent force}

Let us study the radial forces in the absence of currents in the suspension phases. When the rotor is off-centered, the symmetry of the magnetic field in the airgap is broken. This results in a parasitic attraction force between the rotor and the stator. This force has two components acting in the direction of the off-centering $z$ and in the direction perpendicular to it. Assuming that they are proportional to the off-centering, they can be associated with the stiffnesses:

$$
\begin{aligned}
& K_{d, \|}=-\mathfrak{R}\left\{\frac{F}{z}\right\} \\
& K_{d, \perp}=-\mathfrak{J}\left\{\frac{F}{z}\right\},
\end{aligned}
$$

where $\mathfrak{R}$ and $\mathfrak{J}$ denote the real and imaginary parts of their argument. From the model assumptions in section $3, K_{d, \perp}$ is neglected and the value of $K_{d, \|}$ is constant and does not depend on the rotor position. Furthermore, it is assumed that the forces on the rotor in the absence of suspension currents are detent forces only i.e., the impact of the currents flowing in the motor phases is neglected.

Let us validate this by calculating $K_{d, \perp}$ and $K_{d, \|}$ for two different kinds of rotor motion. Figure 3 a corresponds to the static eccentricity configuration, i.e. the rotor spins in a fixed position $z=g / 2$. Figure $3 b$ corresponds to the dynamic eccentricity configuration, i.e. the rotor center whirls around the stator center so that $|z|=g / 2$, while $\omega=0$. In the absence of motor currents, the mean value of $K_{d, \|}$ is $K_{d}=-16.96[\mathrm{kN} / \mathrm{m}]$, whereas the amplitude of $K_{d, \perp}$ does not exceed $4 \%$ of $K_{d}$ for both kinds of rotor motion.

The impact of the motor currents is obtained by setting $I_{A}=-2 I_{B}=-2 I_{C}$, where $I_{A}$ is such that the current density on the copper cross-section of phase A reaches the maximum value of $5\left[\mathrm{~A} / \mathrm{mm}^{2}\right]$. The corresponding results are shown in Fig. 3. In presence of motor currents, the value of $K_{d, \|}$ does not differ by more than $7 \%$ of the value of $K_{d}=-16.96$ $[\mathrm{kN} / \mathrm{m}]$, whereas the amplitude of $K_{d, \perp}$ does not exceed $4 \%$ of $K_{d}$ for both kinds of rotor motion.

In Fig. 3, the stiffnesses vary with the spin and whirl angles. This arises from the interaction between the magnets and the stator saliencies, and the periodicities of the stiffnesses depends on the kind of rotor motion. For more details 


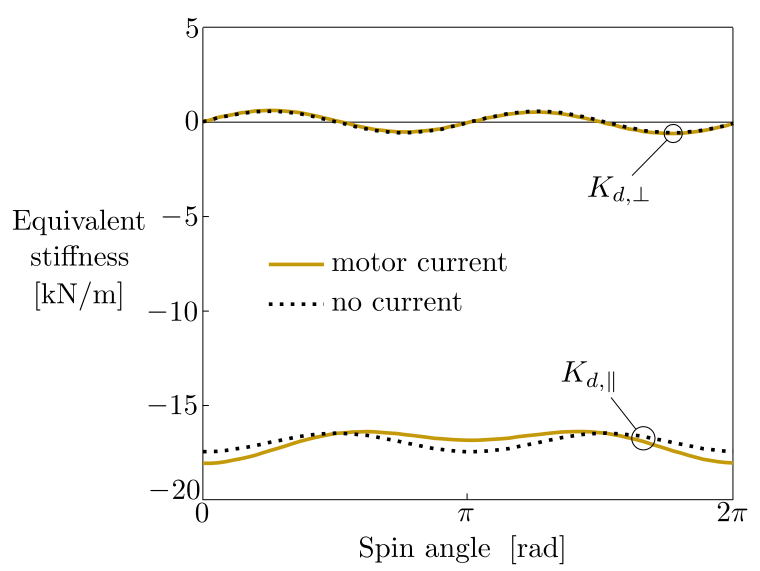

(a)

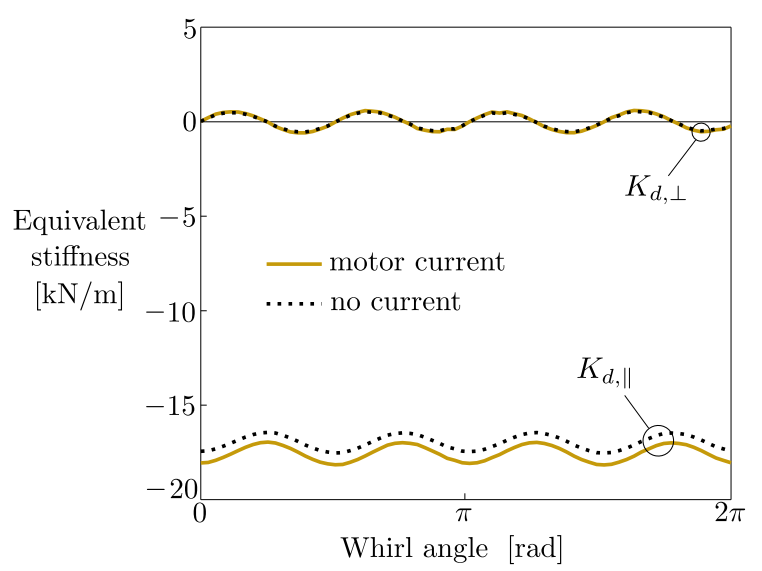

(b)

Fig. 3 Amplitude of the equivalent stiffness between the rotor and the stator in the absence of suspension currents. The motor currents are set to zero (dotted lines) or to maximum values (solid lines) so that $I_{A}=-2 I_{B}=-2 I_{C}$, where $I_{A}$ is such that the current density on the copper cross-section of phase A reaches $5\left[\mathrm{~A} / \mathrm{mm}^{2}\right]$. (a) Data obtained in a static eccentricity configuration. (b) Data obtained in a dynamic eccentricity configuration.

about the study of these effects and the impact of the motor phase currents on the detent stiffness, the reader is referred to (Dorrell et al., 2009).

Finally, other simulations were carried out under the same conditions but with different eccentricities in the range $[g / 10: g / 10: g / 2]$, in presence and in absence of motor currents. They showed that the impact of the eccentricity on $K_{d}$ is small too. In particular, the value of $K_{d, \|}$ does not differ by more than $8 \%$ of the value of $K_{d}=-16.96$ [kN/m], whereas the amplitude of $K_{d, \perp}$ does not exceed $4 \%$ of $K_{d}$, for both kinds of rotor motion.

Considering that an error of less than $10 \%$ is satisfactory in the force prediction, the impact of the off-centering and motor currents on $K_{d, \perp}$ is neglected and $K_{d, \|}$ is assumed to be constant in the next sections: $K_{d, \|} \approx K_{d}=-16.96[\mathrm{kN} / \mathrm{m}]$, and $K_{d, \perp} \approx 0[\mathrm{kN} / \mathrm{m}]$.

\subsection{PM flux linked by the suspension phases}

Let us validate hypothesis 8, which states that only the main PM flux harmonic is considered in the suspension phases, and that it is proportional to the off-centering $|z|$. In this aim, the value of $K_{\Phi}=0.486[\mathrm{~Wb} / \mathrm{m}]$ is identified by evaluating the amplitude of the main PM flux harmonic in the suspension phase A when the rotor spins at $z=g / 4$. This corresponds to the 'fitting curve' in Fig. 4a. As shown in Fig. 4, the PM flux in each suspension phase can then be predicted through the PM flux equation in hypothesis 8. These predictions are then compared to FE results for different amplitudes of the eccentricity $(g / 4$ and $g / 2)$, and for two different kinds of motion.

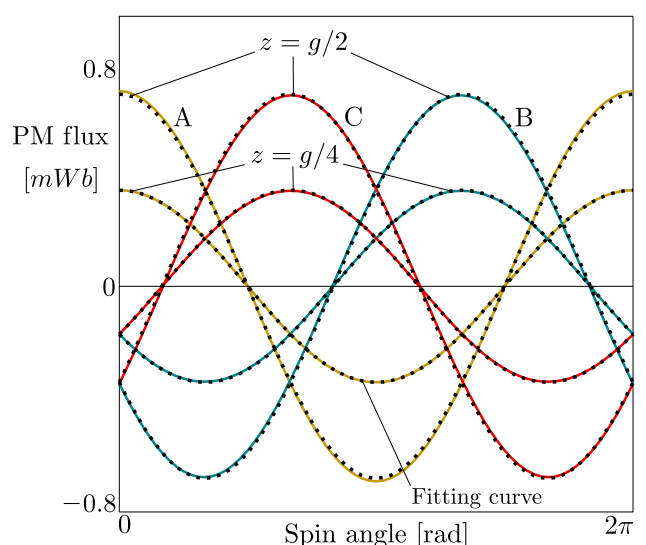

(a)

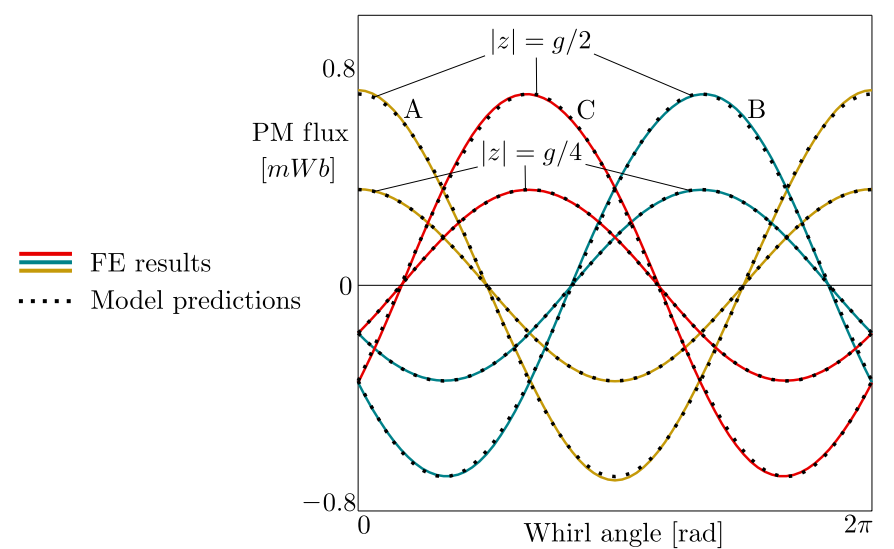

(b)

Fig. 4 Comparison between FE and the model predictions of the PM flux in the suspension phases. The rotor eccentricity is set to $g / 4$ and $g / 2$. Coefficient $K_{\Phi}$ is identified using the data of the 'fitting curve', allowing to predict the PM flux in each winding phase when the rotor (a) spins or (b) whirls, for example. 
Considering the accuracy of the FE method for calculating flux linkages and the close agreement between the predictions from FE and from the model, the previous results validate assumption 8 for the PM flux in the suspension phases.

\subsection{Winding inductances}

Due to the presence of a ferromagnetic shaft, a rotor off-centering impacts the mutual inductances of the suspension windings. Assuming a whirling rotor motion with $|z|=g / 2$, Fig. 5 shows the ratio of the mutual inductances between the suspension phases to their respective average values. The impact of the off-centering on the mutual inductances does not exceed $0.5 \%$ of their average value and is thus neglected.

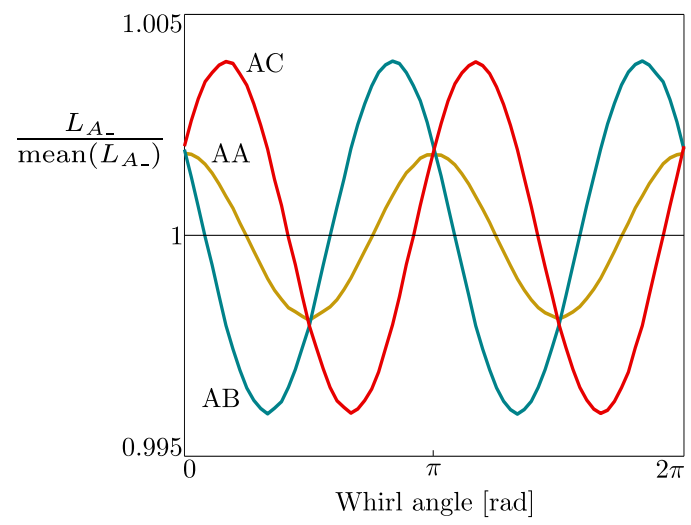

Fig. 5 Ratio of the mutual inductances between the suspension phases to their respective average values.

In conclusion, assumption 6 regarding the inductances is confirmed. From the FE results, the cyclic inductance of the suspension windings is obtained by combining the mutual inductances obtained when the rotor is centered. Assuming a single winding turn, this yields:

$$
L_{c}=L_{A A}-\frac{1}{2} L_{A B}-\frac{1}{2} L_{A C}=32.5[\mu H] .
$$

\section{Forces prediction}

The force on the rotor has two components $F_{\|}$and $F_{\perp}$ that are aligned and perpendicular to the direction of the eccentricity, respectively. Let us calculate these components with the proposed model, using the parameters obtained in the previous section. The accuracy of the model in the static-eccentricity case is then validated by comparing these predictions with FE simulation results.

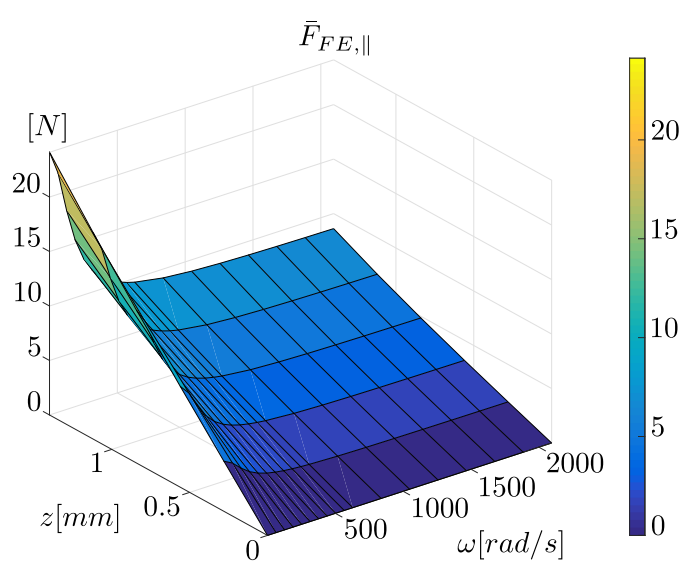

(a)

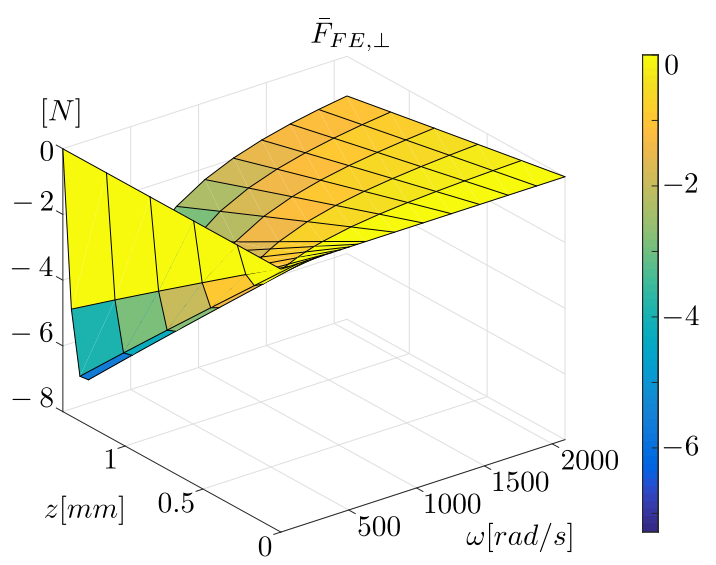

(b)

Fig. 6 (a) and (b) FE predictions of the average forces $\bar{F}_{\|}$and $\bar{F}_{\perp}$ on the rotor spinning in a static eccentricity configuration. 
In this aim, a transient FE model of the motor is run using the following approach. The motor is fed with three-phase balanced sinusoidal currents so that the peak current density in each phase is $5\left[\mathrm{~A} / \mathrm{mm}^{2}\right]$. The force components $F_{\|}$and $F_{\perp}$ are then obtained in a static eccentricity configuration for different spin speeds and eccentricities. Figure $6 \mathrm{a}$ and $\mathrm{b}$ show the average values of $F_{\|}$and $F_{\perp}$ calculated over one full revolution of the rotor. They are denoted $\bar{F}_{\|}$and $\bar{F}_{\perp}$, respectively. As the model is transient, it was run during more than five times the winding time constant $\tau=L_{c} / R$ [s] before recording the data in order to ensure that the suspension currents have reached a steady state.

Let us analyze these results. At zero spin speed, there is no electrodynamic centering force. Only the detent force acts in the direction of the off-centering and its amplitude is given by the curve $\omega=0$ [rad/s] in Fig. 6a. At higher values of $\omega$, the centering electrodynamic force increases and saturates above $\omega=600[\mathrm{rad} / \mathrm{s}]$, because the winding becomes inductive at high speed (Tonoli et al., 2011). The effect is significant, resulting in a reduction of $\bar{F}_{\|}$to $40 \%$ of its value at zero spin speed. Despite this reduction, the electrodynamic force is not sufficient to fully counterbalance the detent force. On the other hand, $\bar{F}_{\perp}$ can also reach significant values of up to $-7.2[\mathrm{~N}]$ at $z=1.4[\mathrm{~mm}]$ and $\omega=189$ [rad/s]. This value decreases at higher speeds. Finally, the model predictions and FE results are compared in Fig. 7a and b, showing the accuracy of the model to predict the centering forces.

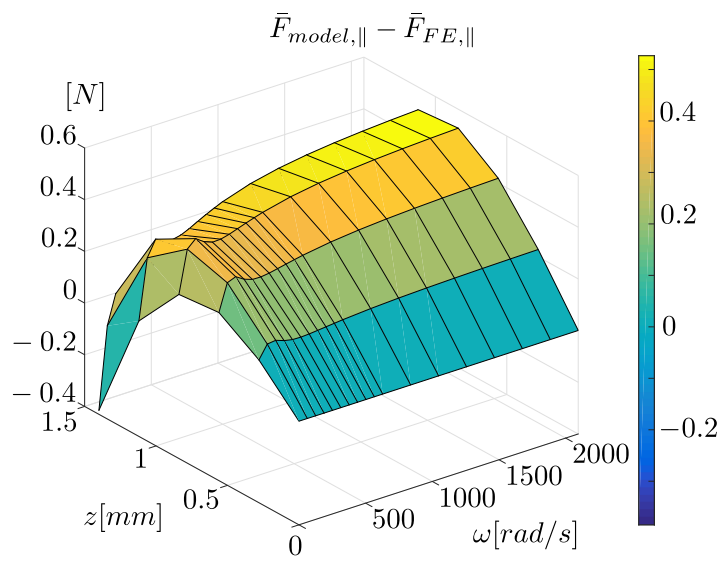

(a)

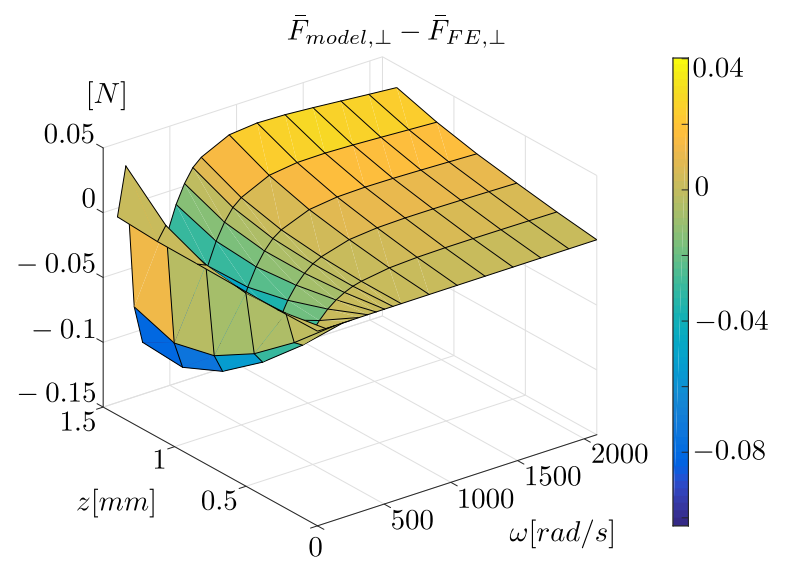

(b)

Fig. 7 Forces on the rotor spinning in a static eccentricity configuration. (a) and (b) Difference between the FE results and the model predictions of the average forces $\bar{F}_{\|}$and $\bar{F}_{\perp}$.

The model predicts that the amplitude of the forces is constant in the static eccentricity configuration. Its predictions fit well to the average values of the forces obtained from the FE simulations, as shown in Fig. 7a and b. However, the amplitude of the forces vary with the angular position of the rotor, according to the FE results. Let us now compare the maximum values of $F_{\|}$and $F_{\perp}$ to their respective average values, in presence of the same motor phase current as for the results shown in Figs. 6 and 7. As shown in Fig. 8a and b, the absolute difference between the maximum and average values of the forces is moderate. In relative terms and for $F_{\|}$, this difference does not exceed $3 \%$ of its average value within the domain shown in Fig. 8a. For $F_{\perp}$, the relative difference remains also low except at low speed and for large eccentricities, where the relative error peaks up because the force amplitude approaches zero.

In particular, let us focus on the result at $(z=1.4 ; \omega=0)$ in Fig. 8a, i.e. about 0.8 [N]. This corresponds to detent forces only and can be compared to the results from Fig. 3a that yielded a maximum difference $F_{\|}-\bar{F}_{\|}$of about 1 [N]. The difference between the two values is relatively small in relation with the average detent force $(0.7 \%)$. This may come from differences between the two FE simulations, the first one being magnetoquasistatic whereas the second one is transient.

Lastly, the average forces $\bar{F}_{\|}$and $\bar{F}_{\perp}$ are calculated in the absence of motor currents and compared to the results obtained previously in presence of motor currents. The difference between the average forces with and without the motor currents are negligible, as shown in Fig. 9a and b. This validates assumption 9 from section 3.

\section{Radial force reduction means}

As shown in the previous section, the off-centering detent force between the magnets and the stator yoke cannot be fully counterbalanced by the centering electrodynamic force. This force is due to the presence of a ferromagnetic yoke at the stator. In order to orient the motor designer toward potential ways of reducing the parasitic effect of the detent force, this section investigates two modifications of the stator. More specifically, the stator teeth thickness and permeability 


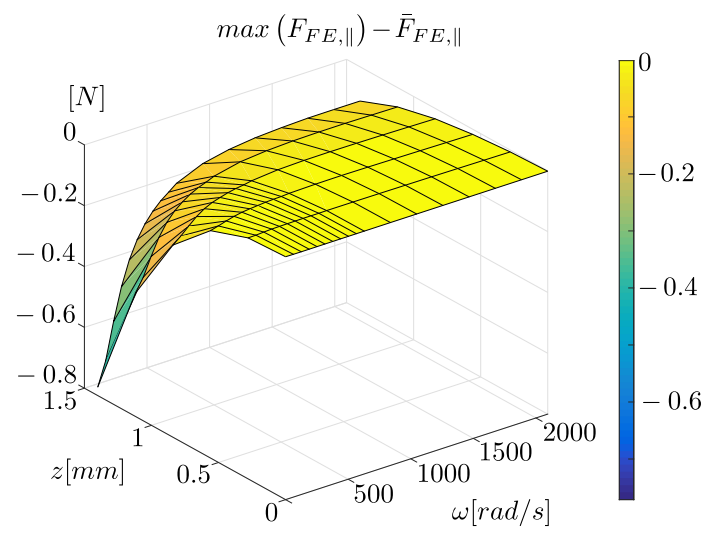

(a)

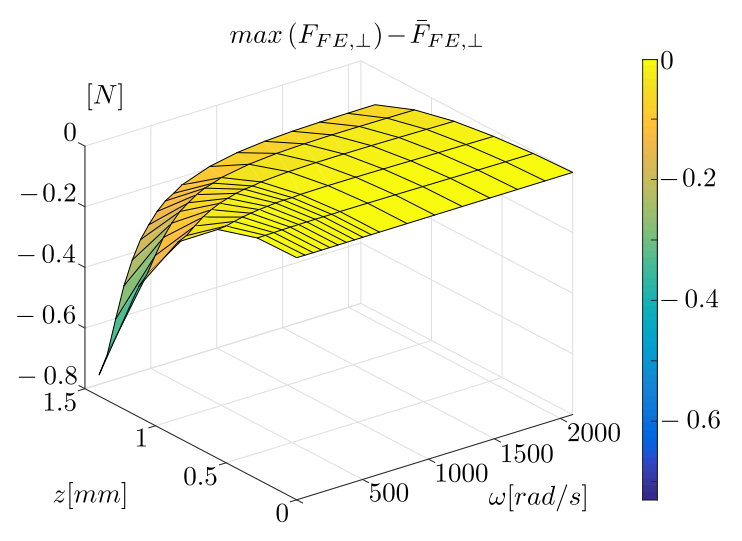

(b)

Fig. 8 Forces on the rotor spinning in a static eccentricity configuration. (a) and (b) Maximum difference between FE predictions of $F_{\|}$and $F_{\perp}$ and their respective average values.

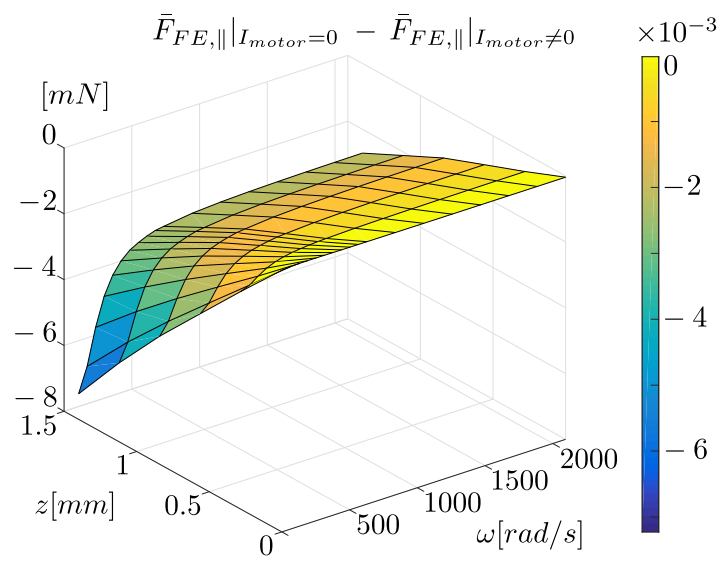

(a)

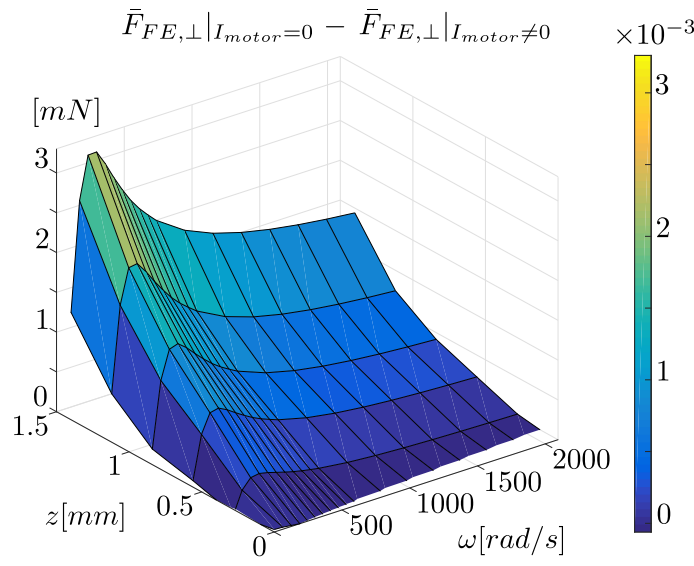

(b)

Fig. 9 Forces on the rotor spinning in a static eccentricity configuration. (a) and (b) Maximum difference between FE predictions of $F_{\|}$and $F_{\perp}$ and their respective average values. (c) and (d) Difference between the average forces on the rotor, with and without motor currents.

are modified, all the other parameters remaining constant. Considering the number of parameters changed, and since the effect of these parameter changes on the motor function and saturation are not considered, this constitutes a preliminary study and no conclusions about optimal design are drawn. However, trends are highlighted, yielding potential ways of reducing the radial forces.

First, let us calculate the radial forces when the stator teeth thickness is modified. The parameters of the model are calculated using the approach presented in section 4. As shown in Fig. 10a, decreasing the teeth thickness has no significant impact on the average force $\bar{F}_{\perp}$ while it has a positive effect on $\bar{F}_{\|}$. On the one hand, the detent component that corresponds to the value of $\bar{F}_{\|}$at zero spin speed increases with the teeth thickness. This corresponds to intuition as the reluctance of the airgap is lower for larger teeth, resulting in a higher PM flux density in the airgap. On the other hand, the absolute value of the electrodynamic component of $\bar{F}_{\|}$slightly decreases with the teeth thickness. In this case, the increase in PM flux linkage is counterbalanced by the higher impedance of the winding. For larger teeth thicknesses, higher values of $R$ and $L_{c}$ then result in lower currents and electrodynamic forces. In conclusion, reducing the teeth thickness decreases the off-centering force, but fully canceling this force requires more drastic changes in the motor design.

A second way to reduce the effect of the detent force consists in changing the permeability of the stator teeth. In this aim, the stator yoke is separated into two parts as shown in Fig. 11, and the teeth relative permeability is set to 1. The forces corresponding to this bearing are shown in Fig. 10b. In this case, their amplitudes are drastically reduced compared to the previous case studies. In particular, the detent force that corresponds to $\bar{F}_{\|}$at zero spin speed is much lower than in Fig. 10a because the ferromagnetic part of the stator yoke is far from the magnets. This increases the airgap reluctance, thereby decreasing the PM flux density. This also reduces the PM flux density in the airgap, resulting in a lower electrodynamic force component. However, this component is high enough to fully cancel the parasitic detent force, so 


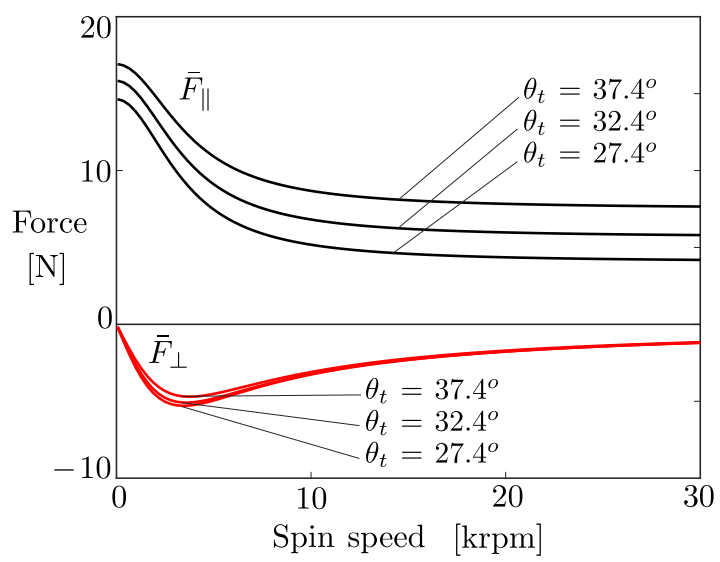

(a)

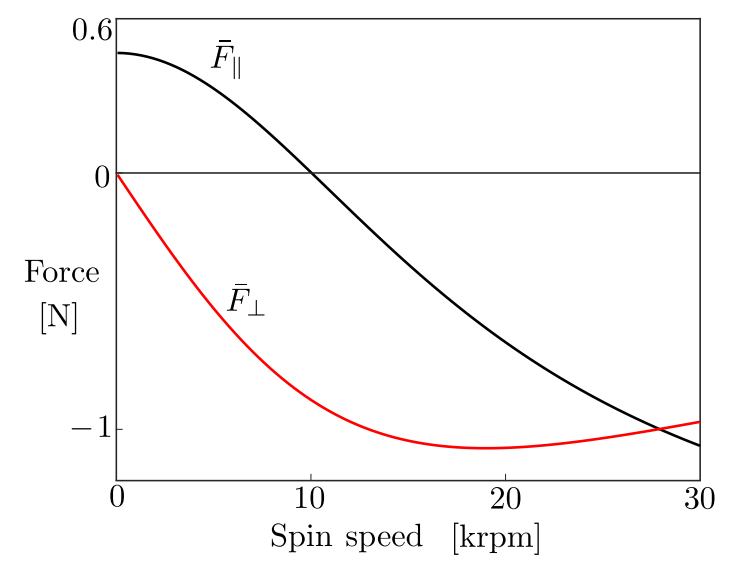

(b)

Fig. 10 Forces on the rotor spinning in a static eccentricity configuration. (a) Ferromagnetic stator teeth with different thicknesses. (b) Non-ferromagnetic stator teeth with $\theta_{t}=32.4^{\circ}$.

that the total force $\bar{F}_{\|}$reaches negative values above $10 \mathrm{krpm}$. Finally, setting the relative permeability of the teeth to 1 does not impact the winding resistance whereas the cyclic inductance is lowered. The winding is thus less inductive and the electrodynamic force saturates at higher speeds, which is reflected in the horizontal position of the curves that is shifted to the right in Fig. 10b.

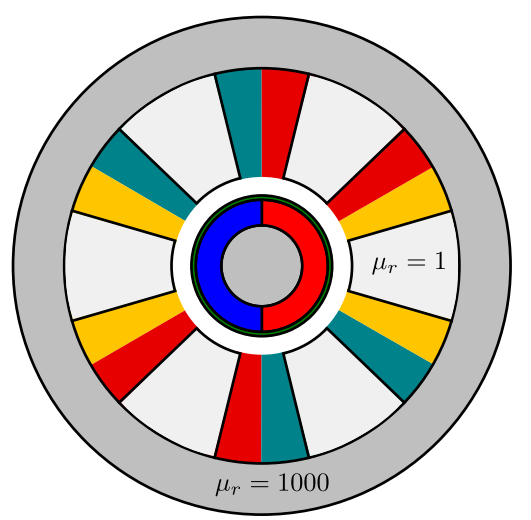

Fig. 11 Bearing with a stator yoke comprising two parts with different permeabilities. The permeability of the teeth is equal to that of air.

In conclusion, decreasing the amount of ferromagnetic material that is close to the PM may be investigated as a means to reduce the radial forces. Of course, this should be done considering the impact of these changes on the motor function simultaneously.

\section{Conclusion}

This paper detailed how the most important assumptions of the EDB model can be validated to show its applicability in the case of a motor with parallel connections in the stator windings. These assumptions are verified for the slotted PM motor that is being designed at the FEMTO-ST institute. In this case, it was shown that the suspension function can be studied separately from the motor function. As a validation of the model, the force predictions were compared with FE simulation results, showing a good agreement between them. In particular, this highlighted the good accuracy of the model at low eccentricities, which corresponds to the most likely operating conditions of the motor.

This study also showed that the electrodynamic force cannot fully compensate the detent force in the present motor. However, the effect of the detent force can be reduced by up to $60 \%$ of its value in the absence of electrodynamic forces. Additional studies were performed to investigate other means of detent force compensation. Changing the width of the stator teeth was shown to have a low impact on the radial forces, whereas setting the teeth relative permeability to unity drastically reduces the radial forces and allows for fully compensating the detent force.

From a theoretical point of view, further studies could include a more detailed analysis of the coupling between the 
suspension and motor functions. Also, other kinds of rotor eccentricities should also be simulated to better illustrate the potential of the model when it comes to predicting the forces for various kinds of rotor motion. From a design point of view, the impact of the off-centering on the copper losses should be quantified, as well as the impact of changing the teeth material on the motor performance.

\section{References}

Burakov, A. and Arkkio, A., Low-order parametric force model for eccentric-rotor electrical machine with parallel connections in stator winding, IET Electric Power Applications, Vol.1, No.4, (2006), pp.592-600.

Burakov, A. and Arkkio, A., Comparison of the unbalanced magnetic pull mitigation by the parallel paths in the stator and rotor windings, IEEE Transactions on Magnetics, Vol.43, No.12 (2007), pp.4083-4088.

Chiba, A., Fukao, T. and Ichikawa, O., Takemoto, M., Dorrell, D. G., Magnetic Bearings and Bearingless Drives (2005), pp.313-315, Newnes.

Dorrell, D. G., Hsieh, M. F. and Guo, Y., Unbalanced magnet pull in large brushless rare-earth permanent magnet motors with rotor eccentricity, IEEE Transactions on Magnetics, Vol.45, No.10 (2009), pp.4586-4589.

Dorrell, D. G. and Ionel, D., Radial forces and vibrations in permanent magnet and induction machines, Proceedings of the IEEE Power and Energy Society General Meeting (2012), pp.1-6.

Dumont, C., Kluyskens, V. and Dehez, B., Null flux radial electrodynamic bearing, IEEE Transactions on Magnetics, Vol.50, No.10 (2014), pp.1-12.

Dumont, C., Kluyskens, V. and Dehez, B., Linear state-space representation of heteropolar electrodynamic bearings with radial magnetic field, IEEE Transactions on Magnetics, Vol.52, No.1 (2016), pp.1-9.

Gilson, A., Tavernier, S., Gerber, M., Espanet, C., Dubas, F. and Depernet, D., Design of a cost-efficient high-speed high-efficiency PM machine for compressor applications, 2015 IEEE Energy Conversion Congress and Exposition (ECCE) (2015), pp.3852-3856.

Kasten, H. and Redemann, C., Influence on unbalanced magnetic pull, Proceedings of the 14th International Symposium on Magnetic Bearings (ISMB14) (2014), pp.758-761.

Kim, U. and Lieu, D. K., Effects of magnetically induced vibration force in brushless permanent-magnet motors, IEEE Transactions on Magnetics, Vol.41, No.6 (2005), pp.2164-2172.

Kluyskens, V., Dumont, C. and Dehez, B., Description of an Electrodynamic Self-Bearing Permanent Magnet Machine, IEEE Transactions on Magnetics, Vol.53, No.1 (2017), pp.1-9.

Li, J. T., Liu Z. J. and Nay, L. H. A, Effect of radial magnetic forces in permanent magnet motors with rotor eccentricity, IEEE Transactions on Magnetics, Vol. 43, No.6 (2007), pp.2525-2527.

Rahideh, A. and Korakianitis, T., Analytical open-circuit magnetic field distribution of slotless brushless permanentmagnet machines with rotor eccentricity, IEEE Transactions on Magnetics, Vol. 47, No.12 (2011), pp.4791-4808.

Tonoli, A., Amati, N., Impinna, F., Detoni, J., A Solution for the stabilization of electrodynamic bearings: modeling and experimental validation. ASME Journal of Vibration and Acoustics, Vol. 133, No.2 (2011), pp.021004-021004-10. 